\title{
Dynamical Effects of Stellar Companions
}

\author{
Smadar Naoz
}

Department of Physics and Astronomy, University of California, Los Angeles, CA 90095, USA

email: snaoz@astro.ucla.edu

\begin{abstract}
The fraction of stellar binaries in the field is extremely high (about $40 \%-70 \%$ for $M>1 \mathrm{M}_{\odot}$ stars), and thus, given this frequency, a high fraction of all exoplanetary systems may reside in binaries. While close-in giant planets tend to be found preferentially in binary stellar systems it seems that the frequency of giant planets in close binaries $(<100-1000 \mathrm{AU})$ is significantly lower than in the overall population. Stellar companions gravitational perturbations may significantly alter the planetary orbits around their partner on secular timescales. They can drive planets to large eccentric orbits which can either result in plunging these planets into the star or shrinking their orbits and forming short period planets. These planets typically are misaligned with the parent star.
\end{abstract}

Keywords. (stars:) planetary systems, (stars:) binaries

\section{Introduction}

Most, if not all, stars are born in binaries or higher multiples and many of them stay in a binary configuration throughout their stellar lifetime $\left(\sim 40-70 \%\right.$ for $\gtrsim 1 \mathrm{M}_{\odot}$, e.g., Raghavan et al. 2010). Thus, it seems likely that a stellar companion will significantly influence the planet formation and evolution in such a system. Furthermore, observations suggest that short period giant planets are more likely to have a far away companion (Knutson et al. 2014;Ngo et al. 2015;Wang et al. 2015). While on the other hand, some studies suggest that the frequency of giant planets in close binaries $(<100-1000 \mathrm{AU})$ is significantly lower than in the overall population (Wang et al. 2015), possibly due to a truncation or influence from the stellar companion.

The general dynamical behavior of multi-planetary systems in stellar binaries can be exceedingly complex and the many body interactions may exhibit highly irregular behavior. Even when in the regime dynamical interactions can be treated secularly, (i.e., orbital average, long term interaction) the system's dynamical evolution can be very complicated. In particular, apsidal precession arising from planet-planet gravitational interactions is in competition with binary companion forced evolution to either suppress or facilitate eccentricity excitations (e.g., Innanen et al. 1997;Takeda et al. 2008;Farr \& $\mathrm{Naoz}$ in Prep.). In light of this phenomenological richness, here we begin by examining the simplest of the unsolved problems - namely, a setup wherein a single planet is perturbed by a binary companion. As will be discussed below, even within the context of this seemingly trivial configuration, complex dynamical evolution associated with pumping of extremely high eccentricities can arise, potentially leading to the production of close-in planetary systems that exhibit spin-orbit misalignments.

In the three-body approximation, dynamical stability requires a hierarchical configuration, in which the inner binary - in our case the primary star and a planet, is orbited by a third stellar companion on a much wider orbit (although we focus on a companion star, it can also be a planet or a brown dwarf). In the latter case, proximity to low-order mean-motion resonances becomes unlikely, the secular approximation (i.e., phase averaged, long term evolution) can be applied. The gravitational potential is then expanded 

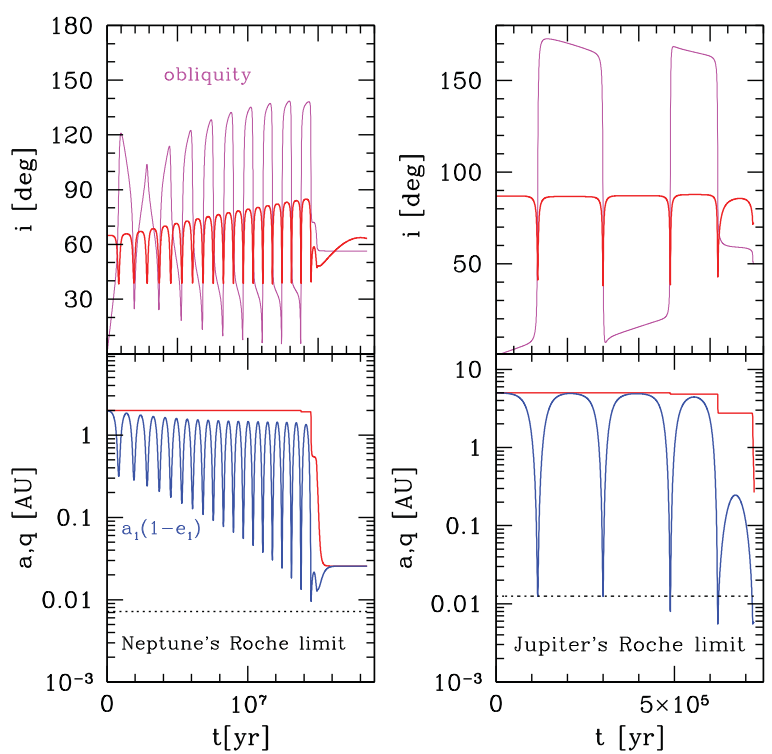

Figure 1. Effects of a companion: Tidal disruption of a planet (right panel) and circularization and shrinking the orbits due to tides (left panel). The systems' mutual inclination (red line), and obliquity (magenta lines) are depicted in the top panels, while the bottom panels shows the semi-major axes (red lines) and pericenter distances (blue lines) in AU. Also shown in dashed lines are the pericenter at which tidal disruption takes place. Left panels show a Neptune-size planet around an $\mathrm{M}$ dwarf $\operatorname{star}\left(0.32 \mathrm{M}_{\odot}\right)$ where a Neptune mass planet is initialized at $a_{1}=2 \mathrm{AU}$, and zero eccentricity. The companion here is a brown dwarf $\left(10 \mathrm{M}_{j}\right)$ at $50 \mathrm{AU}$ with eccentricity of 0.52 . The mutual inclination is set initially to be $65^{\circ}$, and the argument of periapsis of both orbits is set to zero initially. Right panels show a Jupiter mass planet around a sun like star, set initially on a circular orbit at a 5 AU with zero eccentricity. The companion here is a sun like star at at $200 \mathrm{AU}$ with 0.75 eccentricity. The mutual inclination is set initially to be $87^{\circ}$, and the argument of periapsis of both orbits is set to zero initially.

in semi-major axis ratio, $a_{1} / a_{2}$ (Kozai 1962;Lidov 1962), where $a_{1}$ is the semi-major axis of the inner orbit and $a_{2}$ is the semi-major axis of the outer orbit. This ratio is a small parameter due to the hierarchical configuration. The lowest order of approximation, which is proportional to $\left(a_{1} / a_{2}\right)^{2}$ is called the quadrupole-level of approximation.

In early studies of high-inclination secular perturbations, the outer orbit was assumed to be circular and in addition, it was assumed that one of the inner binary members is a massless test particle (Kozai 1962;Lidov 1962). In this situation, the quadrupole-level of approximation, is valid (Naoz et al. 2011,2013a). The resulting Hamiltonian admits a constant of motion that corresponds to the component of the inner orbit's angular momentum along the z-axis (which is parallel to the total angular momentum). Naoz et al. (2011,2013a) showed that relaxing either one of these assumptions, i.e., allowing for an eccentric outer perturber, or going beyond the test particle approximation, leads to qualitative different behavior. This case requires the next level of approximation, called the octupole-level of approximation (Harrington 1968,1969;Ford et al. 2000;Blaes et al. 2002), which is proportional to $\left(a_{1} / a_{2}\right)^{3}$. Accordingly, recent developments in this field have shown that these systems have rich and exciting dynamics (e.g., Naoz et al. 2011,2013a;Lithwick\& Naoz 2011;Katz et al. 2011;Li et al. 2014a).

In the octupole- level of approximation, initially moderate variations in orbital eccentricity can be driven to extremely high values (Ford et al. 2000; Naoz et al. 2013a; Teyssandier et al. 2013; Li et al. 2014a). In addition, the planet's inclination with respect to the 


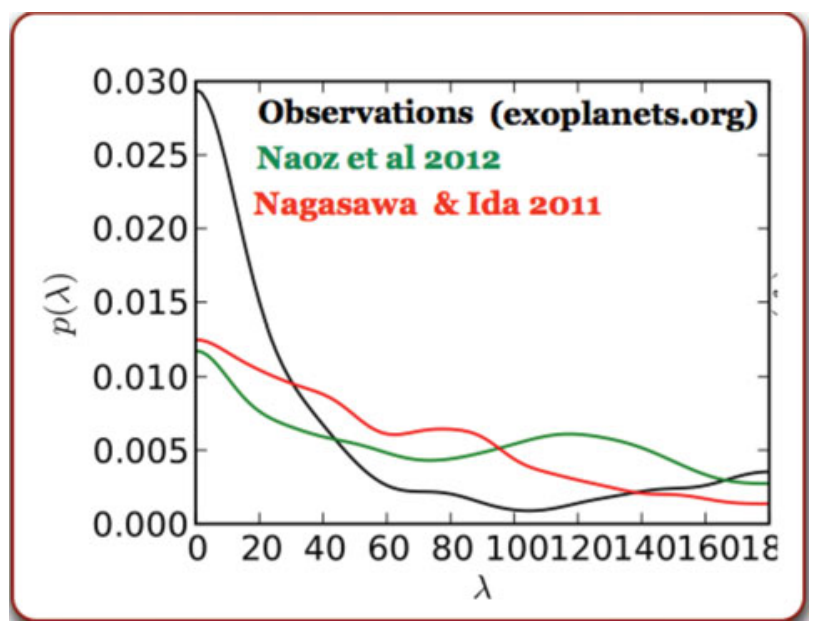

Figure 2. Projected Hot Jupiters obliquity We consider the observed distribution (updated as for 2012, black line) exoplanets.org, and the projected obliquity from Naoz et al. (2012) Monte-Carlo EKL simulations (green line) and planet-planet scattering adopting Nagasawa and Ida 2007, simulations. Bayesian analysis suggests that $\sim 30 \%$ of the population is consistent with EKL formation mechanism. Furthermore, focusing on the misaligned population, the EKL mechanism seems to contributes about $60 \%$ to $80 \%$. Figure adopted from Naoz et al. (2012).

total angular momentum can flip from prograde $\left(<90^{\circ}\right)$ to retrograde $\left(>90^{\circ}\right)(\mathrm{NaOz}$ et al.2011,2013a). This process was coined as the Eccentric Kozai-Lidov (EKL) mechanism, and it was shown that it can be used as a great tool to understand different astrophysical settings.

\section{Gravitational Perturbations from a Companion on the Orbital Configuration of Giant Planets}

As already mentioned above, during the system evolution, the EKL mechanism can induce large eccentricity excitations on the planet's orbit. herefore, on one hand, the small pericenter can lead the planet to tidally disrupt. On the other hand, the tidal forces can shrink and circularize the planets orbit (see Figure 1 left and right panels, respectively). Significant fraction of Jupiters that are initially set at 5 AU stay in their birth place, and undergo moderate eccentricity and inclination oscillations. A small fraction of the systems (which depends on the tidally disrupt distance, e.g., Petrovich 2015 ) survive as Hot Jupiters.

Hot Jupiters formed via gravitational perturbations from a companion star have an interesting consequence: they are likely to be misaligned with their parent star. This was first predicted by Fabrycky and Tremaine (2007) and Wu et al. (2007), for a quadrupolelevel calculation, including GR and tides. The consequence of using the quadrupolelevel is that only a narrow part of the initial configurations is accessible for hot Jupiter generation. In particular, they found that Hot Jupiters are more likely to form in systems orbited by a nearly perpendicular stellar companion (e.g., Fabrycky and Tremaine 2007). Also, for the quadrupole-level to be applicable the companions orbits must be circular (Naoz et al. 2013a).

The EKL mechanism can tap into larger parts of the parameter space which may result in an exciting behavior such as large eccentricity excitations and flipping of the Jupiter orbit with respect to the total angular momentum (e.g., Naoz et al. 2011,2013a;Teyssandier 


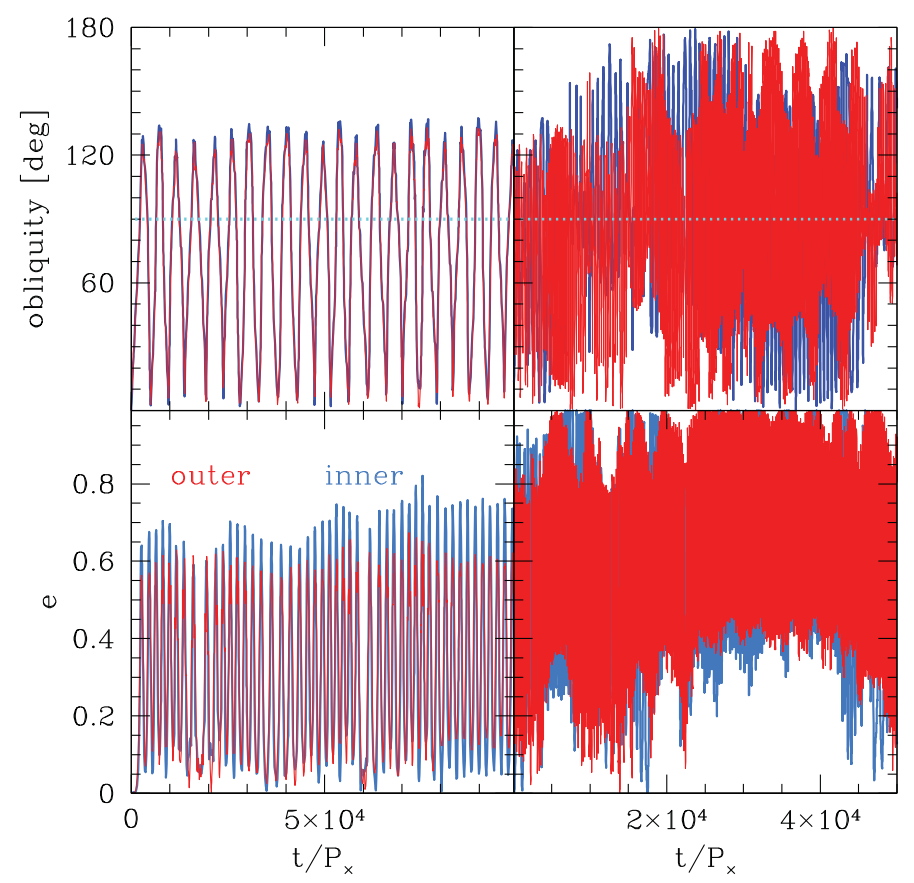

Figure 3. Eccentricity and obliquity evolution for two planet system in the presence of a companion. The left hand panels shows a system for which the outer planet separation is $0.021 \times a_{c}$, where $a_{c}$ is the separation of the companion. The companion eccentricity here is 0.3. The system exhibit stable eccentricity oscillations. The right panels describes a system for which the outer planet separation is $0.06 \times a_{c}$. The companion eccentricity here is 0.5 . In both cases the inner planet is located at half the distance of the outer plant, and the mass ratio is $5 \times 10^{-5}, 3 \times 10^{-5}, 1$ (inner and outer planets to companion, respectively).

et al. 2013; Li et al. 2014a). A Monte-Carlo calculation extended to the EKL mechanism (as well as GR $\dagger$ and tides) showed that indeed the final (and initial) orbital inclinations and obliquity cover large range of the parameter space (Naoz et al. (2012) and Petrovich (2015), and see Figure 2 for the projected obliquity distribution). Furthermore, Naoz et al. (2012) conducted a bayesian analysis assuming that the obliquity distribution is a contribution of three processes, the first is an unknown process which results in an aligned component to the distribution, the second is an EKL mechanism and lastly, a planet-planet scattering process (adopting Nagasawa and Ida 2007). This analysis suggested that a large fraction of the observed high obliquity Hot Jupiter is consistent in forming via the EKL mechanism $\neq$. In particular is seems that about $60 \%$ to $80 \%$ of systems with large obliquities are consisted with EKL mechanism formation scenario. Out of the entire observed population (which has a large aligned component), EKL is consistent with $\sim 30 \%$ of the observed systems while planet-planet scattering contributes about $\sim 10 \%-20 \%$. These results are independent on the formation rates and are based only on the obliquity distribution. The values are consistent with observations that suggest that Hot Jupiters are likely to have a far away companion (e.g., Knutson et al. 2014;Ngo et al. 2015; Wang et al. 2015). However, we note that Ngo et al. 2015 did not find a

$\dagger$ General relativity plays an important role, as it can in some cases suppress eccentricity excitations and in others excite them (e.g., Naoz et al. 2013b).

$\ddagger$ Note that Storch and Lai (2014) and Storch et al. (2015) showed, in the frame work of hierarchical triple systems, that the behavior of the obliquity angle is chaotic and is very sensitive to the stellar spin, assumed here to be $25 \mathrm{~d}$. 
correlation with the misaligned systems. Thus, further investigation requires to explain this discrepancy.

\section{Going Beyond the Three Body System}

As briefly alluded to in the introduction, further phenomenological richness arises when planet- planet interactions are introduced into the picture. We investigate the secular interactions of two planet system in the presence of a stellar companion, using a multibody secular gravitational interaction code. We use the Gauss's averaging method which is a phase-averaged calculation for which the gravitational interactions between nonresonant orbits are treated as being equivalent to angular momentum exchange among massive wires, whose line density in inversely proportional to the orbital velocity (see Touma et al. 2009). The strength of this approach (and thus the code) is that it is not limited to low inclinations and eccentricities or hierarchical configurations. We note however, that the secular approximation breaks if the planets are within few Hill radii of each other, or if they are in mean motion resonances (e.g., Takeda et al.2008). In general this method can be used to explore different many body secular effects, for example the evolution of a particle disk in the presence of a perturber (Batygin 2012).

Figure 3 shows two examples of two planet system evolution in the presence of a stellar companion, considering point mass Newtonian dynamics. These examples have different initial conditions as specified in the caption. The example depicted on the left panels shows a regular time evolution, that exhibits mutual eccentricity oscillations between the orbits. The gravitational forces between the two planets cause the planets angular momenta to precess together around the total angular momentum. Thus, the obliquity angle of each planet (defined here as a tilt angle compare to the initial two planets angular momenta) simply precesses around the inner two angular momenta (known as precession of the nodes), the latter precess around the total angular momentum. Similar behavior was noted in Innanen et al. (1997), and it can eventually produce high obliquity planets (e.g., Li et al. 2014b). The right panels on Figure 3 shows a chaotic evolution for both the eccentricity and the tilt angle of both planets. The two planets react independently to the outer companion gravitational perturbations. Here the system probably becomes unstable. These two examples demonstrate how relatively mild configurational changes in the initial setup of the system can lead to qualitatively different dynamical evolutions.

\section{Acknowledgements}

We thank Konstantin Batygin for thoroughly reading and refereeing the text. S.N. acknowledges partial support from a Sloan Foundation Fellowship.

\section{References}

Batygin, K., 2012, A primordial origin for misalignments between stellar spin axes and planetary orbits Nature, 491, 418-420.

Blaes, O., M. H. Lee, \& A. Socrates, 2002, The Kozai Mechanism and the Evolution of Binary Supermassive Black Holes. ApJ, 578, 775-786.

Ford, E. B., B. Kozinsky, \& F. A. Rasio, 2000, Secular Evolution of Hierarchical Triple Star Systems. ApJ, 535, 385-401.

Harrington, R. S., 1968, Dynamical evolution of triple stars. AJ, 73, 190-194.

—, 1969, The Stellar Three-Body Problem. Celestial Mechanics, 1, 200-209.

Innanen, K. A., J. Q. Zheng, S. Mikkola, \& M. J. Valtonen, 1997, The Kozai Mechanism and the Stability of Planetary Orbits in Binary Star Systems. AJ, 113, 1915. 
Knutson, H. A., B. J. Fulton, B. T. Montet, M. Kao, H. Ngo, A. W. Howard, J. R. Crepp, S. Hinkley, G. Á. Bakos, K. Batygin, J. A. Johnson, T. D. Morton, \& P. S. Muirhead, 2014, Friends of Hot Jupiters. I. A Radial Velocity Search for Massive, Long-period Companions to Close-in Gas Giant Planets. ApJ, 785, 126.

Katz, B., S. Dong, \& R. Malhotra, 2011, Long-Term Cycling of Kozai-Lidov Cycles: Extreme Eccentricities and Inclinations Excited by a Distant Eccentric Perturber. PRL 107, 18.

Kozai, Y., 1962, Secular perturbations of asteroids with high inclination and eccentricity. $A J$, 67, 591.

Lidov, M. L., 1962, The evolution of orbits of artificial satellites of planets under the action of gravitational perturbations of external bodies. planss, 9, 719-759.

Nagasawa, M. and S. Ida, 2011, Orbital Distributions of Close-in Planets and Distant Planets Formed by Scattering and Dynamical Tides. ApJ, 742, 72 .

Li, G., S. Naoz, B. Kocsis, \& A. Loeb, 2014a, Eccentricity Growth and Orbit Flip in Nearcoplanar Hierarchical Three-body Systems. ApJ, 785, 116.

Li, G., S. Naoz, F. Valsecchi, J. A. Johnson, \& F. A. Rasio, 2014b, The Dynamics of the Multiplanet System Orbiting Kepler-56. ApJ 794, 131.

Lithwick, Y. \& S. Naoz, 2011, The Eccentric Kozai Mechanism for a Test Particle. ApJ, 742, 94.

Naoz, S., 2016, The Eccentric Kozai-Lidov Effect and Its Applications. ARAA, submitted.

Naoz, S. \& D. C. Fabrycky, 2014, Mergers and Obliquities in Stellar Triples. ApJ, 793, 137.

Naoz, S., W. M. Farr, Y. Lithwick, F. A. Rasio, \& J. Teyssandier, 2011, Hot Jupiters from secular planet-planet interactions. Nature, 473, 187-189.

—, 2013a, Secular dynamics in hierarchical three-body systems. MNRAS, 431, 2155-2171. .

Naoz, S., B. Kocsis, A. Loeb, \& N. Yunes, 2013b, Resonant Post-Newtonian Eccentricity Excitation in Hierarchical Three-body Systems. ApJ, 773, 187.

Naoz, S., W. M. Farr, \& F. A. Rasio, 2012, On the Formation of Hot Jupiters in Stellar Binaries. $A p J, \mathbf{7 5 4}, \mathrm{L} 36$.

Ngo, H., H. A. Knutson, S. Hinkley, J. R. Crepp, E. B. Bechter, K. Batygin, A. W. Howard, J. A. Johnson, T. D. Morton, \& P. S. Muirhead, 2015, Friends of Hot Jupiters. II. No Correspondence between Hot-jupiter Spin-Orbit Misalignment and the Incidence of Directly Imaged Stellar Companions. ApJ, 800, 138.

Petrovich, C. 2015, Steady-state Planet Migration by the Kozai-Lidov Mechanism in Stellar Binaries. ApJ, 799, 27.

Raghavan, D., H. A. McAlister, T. J. Henry, D. W. Latham, G. W. Marcy, B. D. Mason, D. R. Gies, R. J. White, \& T. A. ten Brummelaar, 2010, A Survey of Stellar Families: Multiplicity of Solar-type Stars. ApJS, 190, 1-42.

Storch, N. I., K. R. Anderson, \& D. Lai, 2014, Chaotic dynamics of stellar spin in binaries and the production of misaligned hot Jupiters. Science, 345, 1317-1321.

Storch, N. I. \& D. Lai, 2015, Chaotic dynamics of stellar spin driven by planets undergoing Lidov-Kozai oscillations: resonances and origin of chaos. MNRAS, 448, 1821-1834.

Takeda, G., R. Kita, \& F. A. Rasio, 2008, Planetary Systems in Binaries. I. Dynamical Classification. ApJ, 683, 1063-1075.

Teyssandier, J., S. Naoz, I. Lizarraga, \& F. A. Rasio, 2013, Extreme Orbital Evolution from Hierarchical Secular Coupling of Two Giant Planets. ApJ, 779, 166.

Wang, J., D. A. Fischer, E. P. Horch, \& J.-W. Xie, 2015, Influence of Stellar Multiplicity On Planet Formation. III. Adaptive Optics Imaging of Kepler Stars With Gas Giant Planets. ApJ, 806, 248. 